\title{
HUMAN DETECTION IN HOURS OF DARKNESS USING GAUSSIAN MIXTURE MODEL ALGORITHM
}

\author{
E.Komagal ${ }^{1}$, V.Seenivasan ${ }^{2}$, K.Anand $^{3}$ and C.P.Anand raj $^{4}$ \\ ${ }^{1-4}$ Department of Electronics and Communication Engg. \\ ${ }^{1-4}$ Velammal College of Engg. And Tech., Viraganoor,Madurai.
}

\begin{abstract}
Video surveillance systems are very important in our everyday life. Video surveillance applications are used in airports, banks, offices and even our homes to remain us secure. Night vision is the ability to see in low light conditions. Surveillance video may not be seen clearly. Especially under the weak illumination conditions. The details of the image are very poor at night. Most of the previous work has focused on daytime Surveillance. This paper proposes a method of Human detection in hours of darkness (night time) using Gaussian Mixture Model (GMM) in real night environment employing an infrared radiation camera. Infrared video is taken as Input to perform Human detection at night. Then the video was processed using Gaussian mixture model algorithm, it was confirmed that by using this method accurate detection of human at night is possible.
\end{abstract}

\section{INTRODUCTION}

Nowadays, crimes, robberies, accidents and terrorist attacks are highly increasing. So it is necessary to develop innovative surveillance systems capable of monitoring very different environments, like buildings (indoor and their neighbours), parking, stores, public parks, galleries, roads,etc. Moving object detection plays a vital role in video surveillance. Most highlevel applications such as abnormal event detection are based on the success of moving object detection. Although a lot of moving object detection methods such as background subtraction have been proposed(1-4). Traditional background subtraction methods perform poorly at night. Robust method is proposed for automatic video surveillance in low-light level environment which has quality problems of low brightness, low contrast and high-level noise. Using this method like illumination compensation and illumination invariant background subtraction to solve the lowquality problem in night surveillance (5). Even though objects in the Retinex image can be recovered clearly and colorfully, Retinex is not suitable to cope with the problem of local illumination changes problem in video sequence. Another preprocessing method for surveillance videos is time-dependent intrinsic images [6] which are regarded as illumination normalized ones. Directional Motion History Images (DMHIs) and Directional Motion Energy Images (DMEIs) for human motion Representation using infrared radiation camera video(7) were also proposed. Park and Jeong [8] use color features to extract candidate regions and then select candidate pixels that may come from signal light from grabbed image based on statistical features. 
International Journal of Information Sciences and Techniques (IJIST) Vol.4, No.3, May 2014

Regions remain are examined to see if each region were of a shape of circle since there are many light sources that have color like signal light. Finally, they remove noise to increase reliability by robust filtering. Pedestrian detection has been analyzed widely in the context of video surveillance with fixed cameras and immobile backgrounds. Techniques like background elimination and analysis, episodic motion, evenness, silhouette shape analysis of the foreground [9], [10], Berrada et al. [11] used simple image subtraction techniques to extract the pixels indicating motion and then computed the mean and standard deviation along the horizontal and vertical axes to identify human motion in a given sequence. In the literature, some researchers focus on detecting vehicles at nighttime [12], traffic lights [13] and tail lights of vehicles in front [14] and human motiondetection and background subtraction (1-9) significant results have been identified by which the existing problems and their solutions are detected.

\section{PROPOSED APPROACH}

Proposed approach for human detection in darkness(night time) is to use the Gaussian Mixture Model(GMM) in real night environment employing an infrared radiation camera. Infrared video is taken as Input to perform Human detection at night. Then the video was used by Gaussian mixture model algorithm in which the Gaussians are used for segmentation of the image. Then the segmented image is subjected to various levels of testing and finally the human beings are identified.

\section{METHODOLOGY}

The GMM chart of the human detection analysis system is shown in Fig.1. Input video here is taken as infrared video and that video is used for processing. For detection of human the processed video is applied for Gaussian mixture model. By this method the foreground (FG) and Background (BGD) of the moving human is extracted. The detected human can also be used for tracking. 


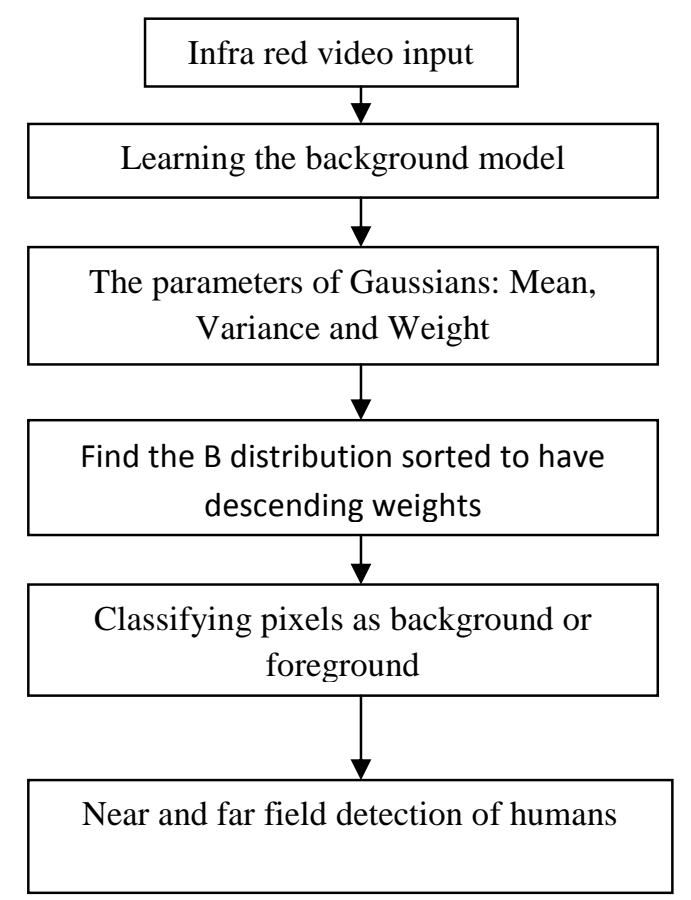

Fig.1.GMM Diagram

\section{MIXTURE OF GAUSSIAN:}

In statistics, a mixture model is a probabilistic model for representing the presence of subpopulations taken as a whole population, without requiring that an observed data set should identify the sub-population. In general a mixture model refers to the mixture distribution that represents the probability distribution of observations in the overall population.

Some ways of implementing mixture models involve steps that attribute postulated subpopulation-identities to individual observations (or weights towards such sub-populations), in which case these can be regarded as types of unsupervised learning or clustering techniques. Inference procedures do not include these steps.

\section{A.LEARNING THE BACKGROUND MODEL}

The sole purpose of using a gaussian mixture model is to obtain accurate segmentation of the target image. The first step in Gaussian mixture model is to learn the background model. The Gaussians are identified and using this the background model is identified.

In the mixture of Gaussians model, parameters of a pixel are modeled as a mixture of Gaussians. Gaussians correspond to the background color is determined. Pixel values that do not fit the 
background distributions are considered foreground until there is a Gaussian that includes them with sufficient evidence behind it. Each pixel of the background is modeled by a separate mixture of ' $K$ ' Gaussians as

$$
P\left(X_{t}\right)=\sum_{i=1}^{k} \omega_{i, t} * \eta\left(X_{t}, \mu_{i}, \sum i, t\right)
$$

where $K$ is the number of Gaussians ( $K=3$ to 5$). X t$ is the present pixel value vector, which consists of red, green, blue component intensity. $\omega_{i, t}$ is an estimate of the weight of the $i^{\text {th }}$ Gaussian in the mixture at time't'; $i_{i, t}$ and $\Sigma_{i, t}$ are respectively the mean value and the covariance matrix of the $\mathrm{i}^{\text {th }}$ Gaussian in the mixture at ' $\mathrm{t}$ '. (This assumes that the red, green, blue pixel components are independent), and ' $\eta$ ' is a Gaussian probability density function.

$$
\begin{gathered}
X_{t}=\left(x_{t}^{r}, x_{f}^{g}, x_{t}^{b}\right) \quad \mu_{i, t}=\left(\mu_{i, t}^{r}, \mu_{i, t}^{g}, \mu_{i, t}^{b}\right) \\
\frac{1}{\left(2 \prod\right) n / 2\left|\sum 1 / 2\right|} e^{-1 / 2}\left(x_{\tau}-\mu_{\tau}\right) \tau * \Sigma-1\left(x_{\tau}-\mu_{\tau}\right)
\end{gathered}
$$

Foreground segmentation consists of two independent harms: 1) estimating the parameters of $\mathrm{k}$ Gaussians and 2) evaluating the likelihood of each Gaussian to represent the background.

\section{B.ESTIMATING PARAMETERS OF K-GAUSSIAN DISTRIBUTIONS}

The weights and means are initialized to 0 . Variances are set to a large value ' $V_{0}$ '. Then at time' $t$ ', every new pixel value ' $X_{t}$ ' is checked against the existing ' $\mathrm{K}$ ' Gaussian distributions, until a match is found. A match is defined as a pixel value ' $\mathrm{X}_{\mathrm{t}}$ ' inside 2.5 standard deviations of a distribution. The ' ' and ' $\Sigma$ ' parameters of the unmatched Gaussian distributions remain the same, and the parameters of Gaussian ' $G_{i}$ ' in the mixture that matches ' $X_{t}^{\prime}$ is updated as follows

$$
\mu_{t}=(1-p) * \mu_{t-1}+\rho * X_{t} \sum_{i, t}=(1-p) * \sum i, t-1+\rho * \operatorname{diag}\left[\left(x_{t}-\mu_{i, t}\right)^{\tau} *\left(x_{t}-\mu_{i, t}\right)\right]
$$

Where $\rho=\alpha^{*} \eta(X t \mid i, t-1, \Sigma i, t-1)$, ' $\alpha$ ' is the learning rate, diag [x] produces a diagonal matrix from matrix ' $X_{t}^{\prime}$ '. If none of the ' $K$ ' Gaussians matches the current pixel value ' $X_{t}^{\prime}$ ', the least probable distribution $G j$ is reassigned, where

$j=\arg \min \{\omega i, t-1\}, \omega j, t-1=w 0, \mu j t=x t$,

$$
\sum j, t-1=v_{0}, l
$$

Where, ' $W O$ ' is a small prior weight; ' $T$ ' is a $3 \times 3$ identity matrix. Then the weight of all ' $k$ ' Gaussian distributions at time't',' $\omega_{i, t}$ ' are updated as:

$$
\omega_{i, t}=(1-\alpha)^{*} \omega_{i, t-1}+\alpha^{*} m_{i, t} \quad \omega_{i, t}=\omega_{i, t} / \sum_{m=1}^{k} \omega_{i, t}
$$


Where, ' $M_{i, t}=1$ ' for the Gaussian distribution, ' $G_{i}$ ' which matched the ' $X_{t}^{\prime}$, and for the unmatched Gaussians.

\section{BACKGROUND MODEL ESTIMATION}

After the parameters of each pixel model are updated, the Gaussians which are most likely to be produced by background processes are determined. First, the Gaussians are ordered by the value of $\omega$, so the most likely background distributions will remain on top and the less probable transient background distributions will move towards the bottom and eventually be replaced by new distributions. Then, the first ' $B$ ' distributions are chosen as the background model and expressed as shown in equation.

$$
B=\arg \min { }_{b}\left(\sum_{m=1}^{k} \omega_{k}>T\right)
$$

Where ' $\mathrm{T}$ ' is a threshold $(0.5<\mathrm{T}<1)$, the first ' $B$ ' components of the sorted mixture Gaussians are responsible for the background. If the pixel ' $X t$ ' is best modeled by one of the background components (the pixel value ' $X t$ ' matches one of the ' $B$ ' distributions), it is marked as background, and otherwise it is classified as foreground.

\section{RESULTS AND DISCUSSION}

To evaluate the Human detection in environment at night by using MATLAB 2010.Here Infrared Video was extracted as sequences and these sequence is used GMM algorithm to obtain the accurate results of human detection and tracking The results are shown below Fig.(2)

Infrared video dataset is taken from OTCVBS and the segmentation is obatined by the software MATLAB R2010A.The challenges faced during the detection of human beings includes detecting the far field humans and contour detection. The best output was achieved by this method and slight distortions have been significantly reduced. 


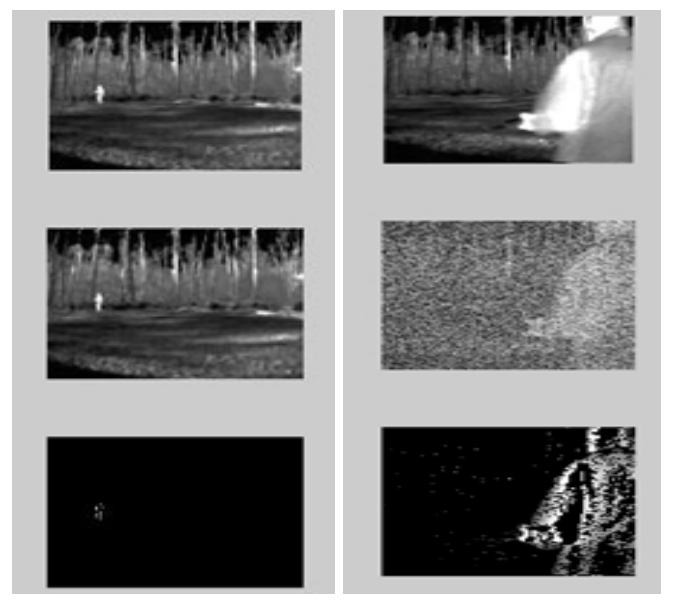

Fig 2. Detected near and far field outputs

\section{CONCLUSION}

Human moving object detection at night is a very important focus for video scrutiny. A lot of particular measures often are taken place in the night condition, for happening, the robbery of resources. Unfortunately, traditional technique only works with sufficient intensity situation. This is because that the challenges of low contrast and high-level noise usually accompany with low brightness condition. The significant variation of illumination from narrow brightness source is another huge challenge. Surveillance video may not be seen visibly. specially under the weak illumination conditions. the image detail of the night video is very poor. Most of the previous work has focused on daytime Surveillance. This paper proposes a method of Human detection in hours of darkness(night time) using GMM algorithm in real night environment employing an infrared radiation camera. infrared video is taken as Input to perform Human detection at night. Then the video was used by GMM GMM algorithm, it was confirmed that by using this method performs accurately to detect human at night is possible. The research show that our method can really detect moving object at night accurately.

\section{REFERENCE}

[1] C. Stauffer and W. E. L. Grimson, "Learning patterns of activity using real-time tracking," IEEE Trans. on Pattern Analysis and Machine Intelligence, vol. 22, no. 8, pp. 747-757, 2000.

[2] P. KaewTrakulPong and R. Bowden, "A real time adaptive visual surveillance system for tracking low-resolution colour targets in dynamically changing scenes," Journal of Image and Vision Computing., vol. 21, no. 10, pp. 913-929, Sep. 2003.

[3] J. H. Duncan and T.C. Chou, "On the detection of motion and the computation of GMM GMM," IEEE Trans. Pattern Analysis and Machine Intelligence, vol. 14, no. 3, pp. 346-352, Mar. 1992.

[4] C. Braillon, C. Pradalier, J. L. Crowley, and C. Laugier, "Real-time moving obstacle detection using GMM GMM models," IEEE Intelligent Vehicles Symposium, Tokyo, Japan, Jun 13-15, 2006, pp. 466-471. 
International Journal of Information Sciences and Techniques (IJIST) Vol.4, No.3, May 2014

[5] Yuan-Kai Wang, "Moving Object Detection for Night Surveillance", 2010 Sixth International Conference on Intelligent Information Hiding and Multimedia Signal Processing, 2010 IEEE DOI 10.1109/IIHMSP.2010.66.

[6] Matsushita, K. Nishino, K. Ikeuchi, and M. Sakauchi, "Illumination normalization with timedependent intrinsic images for video surveillance," IEEE Trans. on Pattern Analysis and Machine Intelligence, vol. 26, no. 10, pp. 1336-1347, Oct. 2004

[7] Tooru Hiroshima, Joo Kooi Tan, Hyoungseop Kim, Seiji Ishikawa, "Human Motion Recognition Employing Infrared Radiation Camera Images", SICE Annual Conference 2012 August 20-23, 2012, Akita University, Akita, Japan,PP:392-395.

[8] J. H. Park, C. S. Jeong, "Real-time Signal Light Detection," Proc. Future Generation Communication and Networking Symposia, 2008.

[9] Haritaoglu et al., "Backpack: Detection of people carrying objects using silhouettes," presented at the Int. Conf. Computer Vision, Corfu,Greece, Sept. 1999.

[10] I. Haritaoglu, “W4: Real-time surveillance of people and their activities,"IEEE Trans. Pattern Anal. Machine Intell., vol. 22, pp. 809-831,Aug. 2000.

[11] D. Berrada , M. Romero , G. Abowd , M. Blount , J. Davis, "Automatic administration of the Get Up and Go Test," Proceedings of the 1st ACM SIGMOBILE international workshop on Systems and networking support for healthcare and assisted living environments, San Juan, Puerto Rico, June 11, 2007.

[12] Y. L. Chen, Y. H. Chen, C. J. Chen,and B. F. Wu, “Nighttime vehicle detection for driver assistance and autonomous vehicles," Proc. IEEE ICPR, pp. 687-690,2006.

[13] J. H. Park, C. S. Jeong, "Real-time Signal Light Detection," Proc. Future Generation Communication and Networking Symposia, 2008.

[14] R. O'Malley, M. Glavin, E. Jones, “Vehicle Detection at Night Based on Tail-Light Detection,"Proc. International Symposium on Vehicular Computing Systems, 2008. 